\title{
TORRE MARIMON: UNA PEQUEÑA CUENCA HIDROLÓGICA EN EL MEDIO AGRARIO MEDITERRÁNEO
}

\author{
R. JOSA MARCH \\ A. HERETER QUINTANA \\ Escola Superior d'Agricultura de Barcelona. UPC. c/ Urgell 187. 08036 Barcelona
}

C. electrónico: Ramon.Jose $@$ upc.es

RESUMEN: Se presenta la cuenca experimental de Torre Marimon (CETM), en primer lugar mediante los trabajos realizados para su caracterización (incluida la cartografía de parámetros edáficos) que permiten valorar su interés. A continuación se hace referencia a los principales resultados obtenidos: balance bídrico; erosión y degradación del suelo asociado al uso; efectos del sistema de laboreo en relación con el contenido de materia orgánica y a la disponibilidad de nutrientes. La cuenca lleva en funcionamiento desde 1993.

ABSTRACT: In this paper the characteristics of the experimental catchment of Torre Marimon (CETM) are defined (including the edaphic cartography). Next the autbors analyze some results obtained: bydric balance, soil erosion in relation to the soil uses, effects of agricultural technics in relation to the organic matter contents and the availability of nutrients. The catchment has funtioned since 1993.

Palabras clave: Cuenca agrícola, Hidrología, Suelos, Erosión, Laboreo de conservación.

Key word: Farmed catchment, Hydrology, Soils, Erosion, Conservation tillage systems.

\section{Introducción}

El interés de instrumentar la cuenca de Torre Marimon (CETM) surgió por la confluencia de diversas motivaciones. Por una parte, colaboraciones anteriores dirigidas a caracterizar los suelos de cuencas no agrícolas del Pirineo y Prepirineo (Vallcebre, por ejemplo) facilitaron el acceso a técnicas de medida de los parámetros necesarios para el funcionamiento de los modelos hidrológicos. Como extensión de estos trabajos surgió el interés en trasladar algunas de estas técnicas al ámbito agrícola para evaluar las transformaciones periódicas que experimenta el suelo a consecuencia del laboreo y su influencia en los procesos erosivos y de gestión de la reserva hídrica.

Existía una amplia bibliografía sobre las relaciones entre el comportamiento hidrológico y el uso del suelo, especialmente asociado a la textura. Pero la progresiva aplicación de prácticas de laboreo de conservación y en algunos casos el abandono de cultivos que favoreció en su momento la PAC, motivó un renovado interés por evaluar los efectos diferenciales inducidos en una cubierta edáfica por el uso del suelo y los sistemas de laboreo. Desde la óptica de la conservación de suelos la 
medida de estas transformaciones en áreas control podía permitir la posterior generalización, y complementar así las recomendaciones sobre la aplicación de uno u otro sistema de laboreo.

El desarrollo de esta línea de trabajo requería un marco estable donde poder llevar a cabo experiencias controladas de laboreo, con fácil accesibilidad y la posibilidad de intervenir de forma muy ágil en la gestión agrícola a fin de disponer del registro y el control de las intervenciones que requiere el proceso productivo. El emplazamiento de la cuenca, en su origen se vio favorecido por la accesibilidad a la finca de Torre Marimon, propiedad de la Diputación de Barcelona, parte de la cual se venía utilizando como área de trabajos prácticos. La localización de la cuenca se presenta en la Fig. 1.

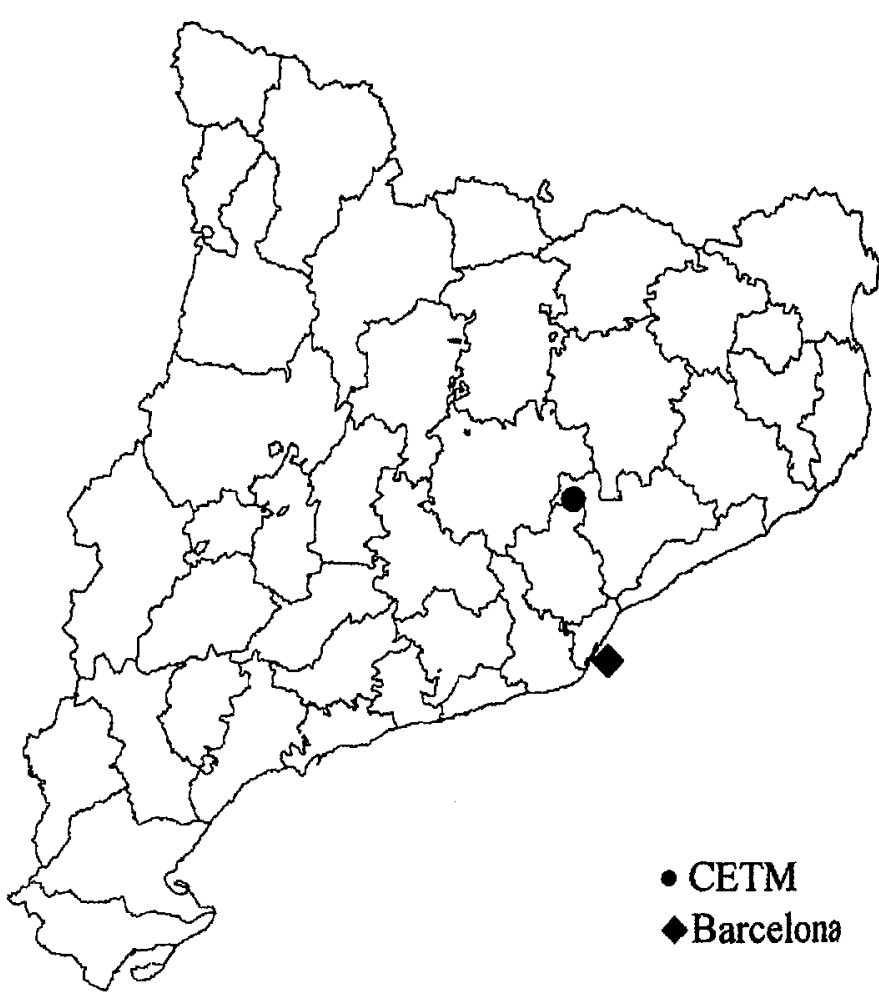

Figura 1. Mapa general de situación de la CETM.

\section{La cuenca y su entorno.}

Debía verificarse la idoneidad de la cuenca desde el punto de vista de su significación en el contexto geográfico. Se consideraron tres factores esenciales: los usos actuales del suelo y su relación con el resto de la comarca, la representatividad edáfica en relación con su entorno inmediato, y en concreto con la llamada Depresión del Vallès, y por último debía establecerse la caracterización climática dentro del ámbito mediterráneo.

Tabla 1. Datos comparativos de los usos del suelo a escalas regional y local

\begin{tabular}{lcccc}
\hline Usos del suelo & Catalunya & Vallès Oriental & Caldes de M. & CETM \\
\hline Superficie forestal (\%) & 61 & 68 & 60 & 3 \\
Superficie labrada (\%) & 33 & 15,5 & 37 & 95 \\
Urbano (\%) & 5 & 16 & 3 & 0 \\
Aguas (\%) & 0,8 & 0,5 & sd & 2 \\
Superficie agraria (ha) & 2471590 & 59332 & 1833 & 27 \\
Superficie total (ha) & 3190110 & 85190 & 3790 & 27 \\
\hline
\end{tabular}

sd= Sin determinar. Fuente: Censo agrario de 1989. (G.C., 2000). Página web del IEC: http://idescat.es (15/01/01).

En la tabla 1 se presentan algunos datos comparativos de usos del suelo en el ámbito geográfico de Catalunya, de la comarca del Vallès Oriental y del término 
municipal de Caldes de Montbui, así como los de la propia cuenca. Se trata, de acuerdo con los objetivos iniciales, de una cuenca agrícola emplazada en una comarca que recibe una fuerte influencia directa del núcleo urbano barcelonés, lo que provoca una fuerte competencia entre la actividad tradicional (agricultura) y el desarrollo industrial, hecho reflejado en las diferencias de ocupación urbana del territorio. A escala de término municipal y en relación con Catalunya, se mantiene una proporción similar entre usos agricola, forestal y urbano.

En la comarca, el principal uso del suelo es el forestal (Tabla 2) formado por una mezcla de planifolios y de resinosas. En la cuenca está representada por una pequeña masa forestal de pinos carrasco y piñonero (según Verdú, 1999) plantada presumiblemente entre 1940 y 1958 . Hay testimonios de la vegetación climácica de la zona que es de tipo encinar litoral (Quercetum ilicis galloprovinciale). En cuanto a usos agrícolas del suelo, en la CETM están representados los principales cultivos tradicionales del mediterráneo (Tabla 3) y de la agricultura extensiva. Sin embargo, no están incluidas las tendencias más recientes relacionadas con el cultivo de ornamentales y otros herbáceos que se introducen principalmente gracias al incremento del número de invernaderos.

Tabla 2. Distribución de especies forestales en la comarca $y$ en el término municipal (superficies en ba)

\begin{tabular}{rccc}
\hline & Catalunya & Vallès Oriental & Caldes de M. \\
\hline Planifolios & 218848 & 13474 & 102 \\
Resinosos & 549898 & 8770 & 990 \\
Mixto & 212617 & 18285 & 5 \\
Total & 981364 & 40525 & 1097 \\
\hline
\end{tabular}

Fuente: Censo agrario de 1989, (G.C., 2000). Página web del IEC:

http://idescates $(15 / 01 / 01)$.

Tabla 3. Distribución de superficies de los cultivos en la CETM y en el término municipal de Caldes de $M$.

\begin{tabular}{lcc}
\hline Cultivos & T. Municipal (*) & CETM $\left(^{* *}\right)$ \\
\hline Olivo (\%) & 4 & 9 \\
Viña (\%) & 2 & 14 \\
Cereal grano (\%) & 31 & 16 \\
Forraje (\%) & 28 & 33 \\
Leguminosas (\%) & 2 & 17 \\
Herbáceos y ornamental (\%) & 29 & 0 \\
Barbecho (\%) & 2 & 1 \\
Otros (\%) & 2 & 10 \\
Superficie total (ha) & 468 & 24.5 \\
\hline
\end{tabular}

Fuentes: * Censo agrario de 1989. (G.C., 2000).

* Corresponden al ciclo agricola 1998-99 (Gomila,J.M., 2000). 
La cubierta edáfica a escala comarcal, esta constituida fundamentalmente por la asociación de Calcic cambisole, con otros suelos como Calcaric phaeozem, Rendzinas, Lithosoles y Calcaric regosoles (C.E.C., 1985). Localmente estas asociaciones se presentan con características más complejas y ligadas a la litología local predominante.

De hecho, en un estudio realizado por Playà Rabella (1998) que recoge la diferenciación clásica de tres unidades geográficas consecutivas (Sierra Litoral, Depresión Prelitoral, donde se ubica la CETM, y Sierra Prelitoral) reconoce en la unidad central la presencia de hasta 12 unidades litoestratigraficas distintas (I.T.G.E., 1993) cada una de las cuales tienen una extensión de más de 1000 ha y de las cuales cinco son las predominantes ocupando cerca del $90 \%$ de la superficie de la unidad central (Tabla 4).

La finca Torre Marimon está situada entre el Mioceno superior (arcillas y areniscas arcósicas) y el Cuaternario (fundamentalmente aluvial indiferenciado). La CETM está íntegramente en la primera de estas dos unidades. Litológicamente se observa la presencia de las arcillas fuertemente edafizadas en la mayor parte de la cuenca y sólo en el extremo NW se observan áreas de arenas con una mineralogía que manifiesta su origen local (atribuible a un afloramiento granítico de Caldes de Montbui). En una prospección geoeléctrica llevada a cabo en la cuenca (Casas et al., 1999) se puede ver como hay una secuencia que, desde el techo a la base incluye: arcillas y un lentejón de material limoso y otro paquete inferior de arcillas (Fig. 2). Por último parece existir un nivel de gravas y arenas con gravas.

\section{GEOELECTRICAL PROFILES AT TORRE MARIMÓN CATCHMENT}
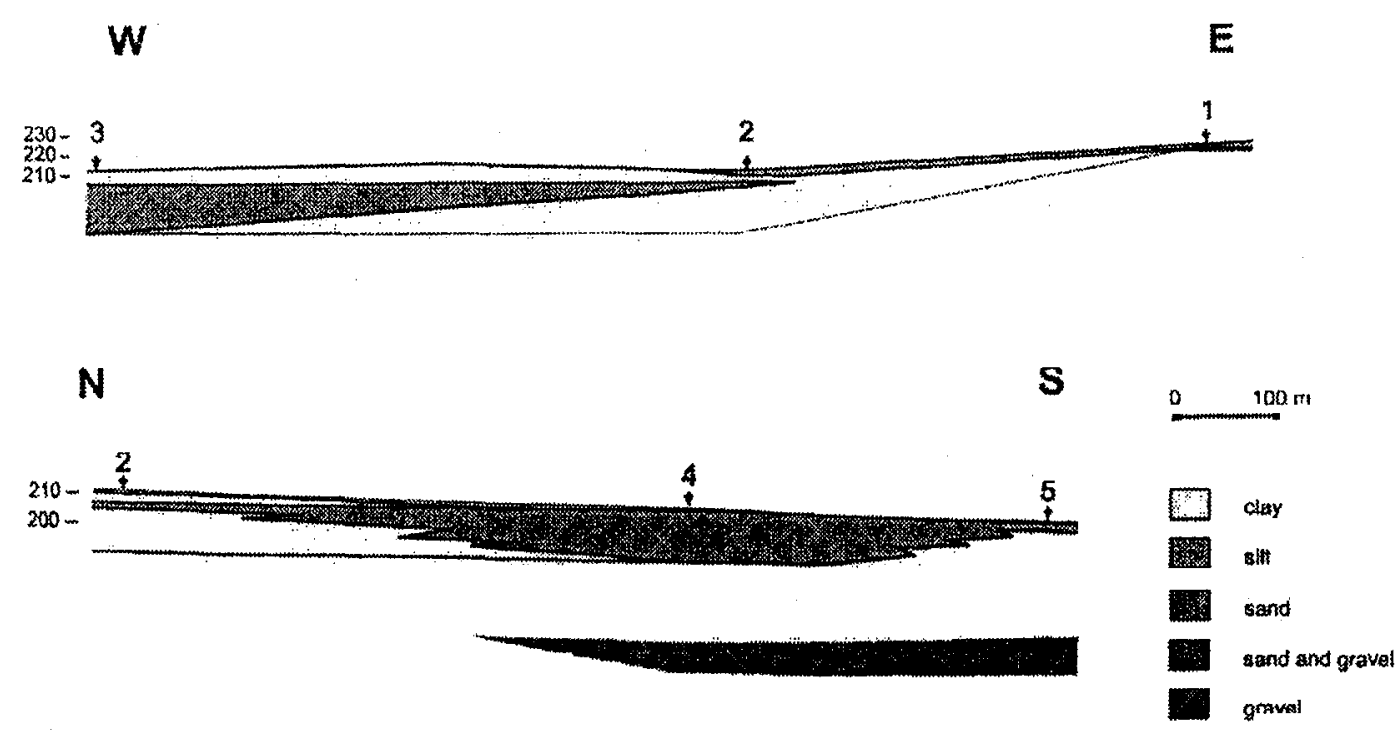

Figura 2. Perfiles geoeléctricos de la cuenca de Torre Marimon (Fuente: Casas et al., 1999)

Es de destacar el aspecto asimétrico del tramo superficial de la cuenca ya que la vertiente NE es más rica en limos que la vertiente SW. La existencia de un potente paquete de arcillas y de limos asegura la impermeabilidad de la cuenca y por tanto su estanqueidad subsuperficial. No obstante, cabe señalar que la experiencia ha demostrado que la red de caminos existente comporta pérdidas de escorrentía, al reconducir el drenaje de alguna pequeña área directamente hacia el exterior de la cuenca. 
Climáticamente la cuenca pertenece al ámbito mediterráneo seco. En la tabla 5 se recoge la clasificación climática tal como la presenta Queralt (1995). Como sucede en toda el área mediterránea, la irregularidad intermensual e interanual es muy elevada y, a pesar de la existencia de un relativamente importante déficit hídrico anual, también hay años húmedos y se producen precipitaciones muy intensas, de forma que los fenómenos erosivos parecen más asociados a estos eventos de periodicidad superior a la anual, que no a un proceso erosivo continuo. En la Fig. 3 se presenta el periodo de retorno de las precipitaciones máximas en 24 horas, para el área de Caldes de Montbui (D.G.C., 2000).

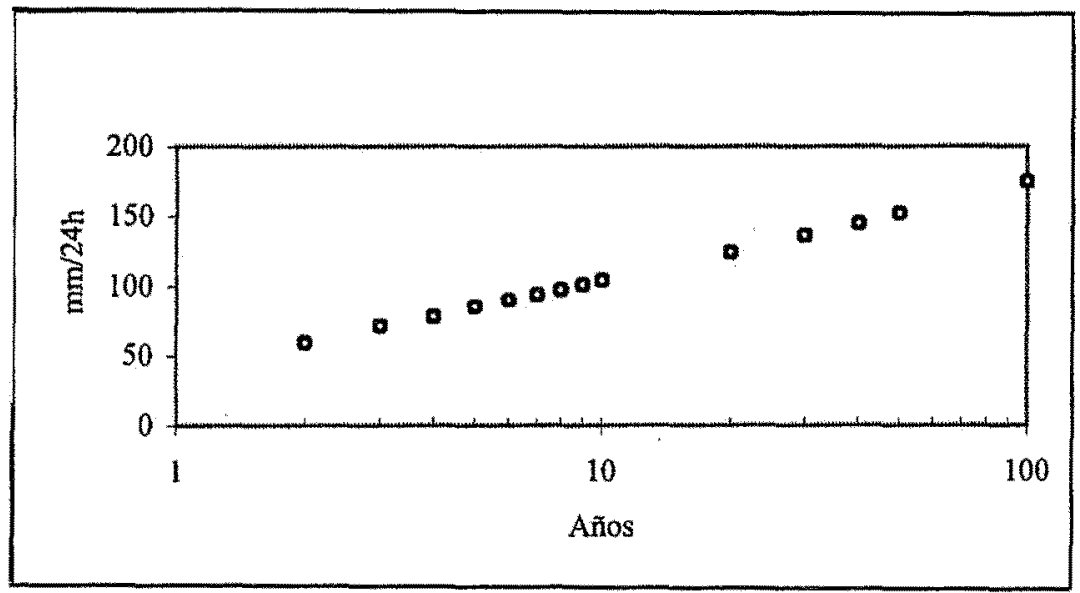

Figura 3. Periodo de retorno de precipitaciones máximas en 24 boras.

Tabla 4. Principales materiales geológicos del sector central de la comarca del Vallès Oriental

\begin{tabular}{llc}
\hline \multicolumn{1}{c}{ Periodo } & \multicolumn{1}{c}{ Unidades Litoestratigraficas } & Superficie $\left(\mathrm{km}^{2}\right)$ \\
\hline & Granodiorita & 28.5 \\
\hline Mioceno medio & Arenisca, limolita y conglomerados & 26.5 \\
y superior & Arcillas y lentejones de arena y conglomerados & 28.5 \\
& Arcillas y areniscas arcósicas & 95.5 \\
\hline Cuaternario & Aluvial indiferenciado, piedemonte cuaternario antiguo & 67.35 \\
\hline
\end{tabular}

Fuente: Playà Rabella,T., (1998) y ITGE (1993).

Tabla 5. Características climáticas del Vallès Occidental, correspondientes a la estación de Torre Marimon

Precipitación media anual: $616 \mathrm{~mm}$

Temperatura media anual: $14.7^{\circ} \mathrm{C}$

Evapotranspiración potencial anual (Thornthwaite): $787 \mathrm{~mm}$

Régimen de humedad

Régimen térmico

Régimen de humedad

Régimen térmico
Clasificación según el criterio de Thornthwaite

Húmedo con falta intensa de agua en verano Mesotérmico, con evapotranspiración estival relativamente baja

\section{Clasificación según el criterío de Papadakis}

Mediterráneo seco

Templado caluroso 


\section{Características de la cuenca e instrumentación}

La caracterización hidrológica de la cuenca así como su instrumentación se recogen en Cañameras et al. (1998) y un resumen de las principales características de la cuenca se presentan en la tabla 6. En la Fig. 4 se presenta un MDT de la cuenca.

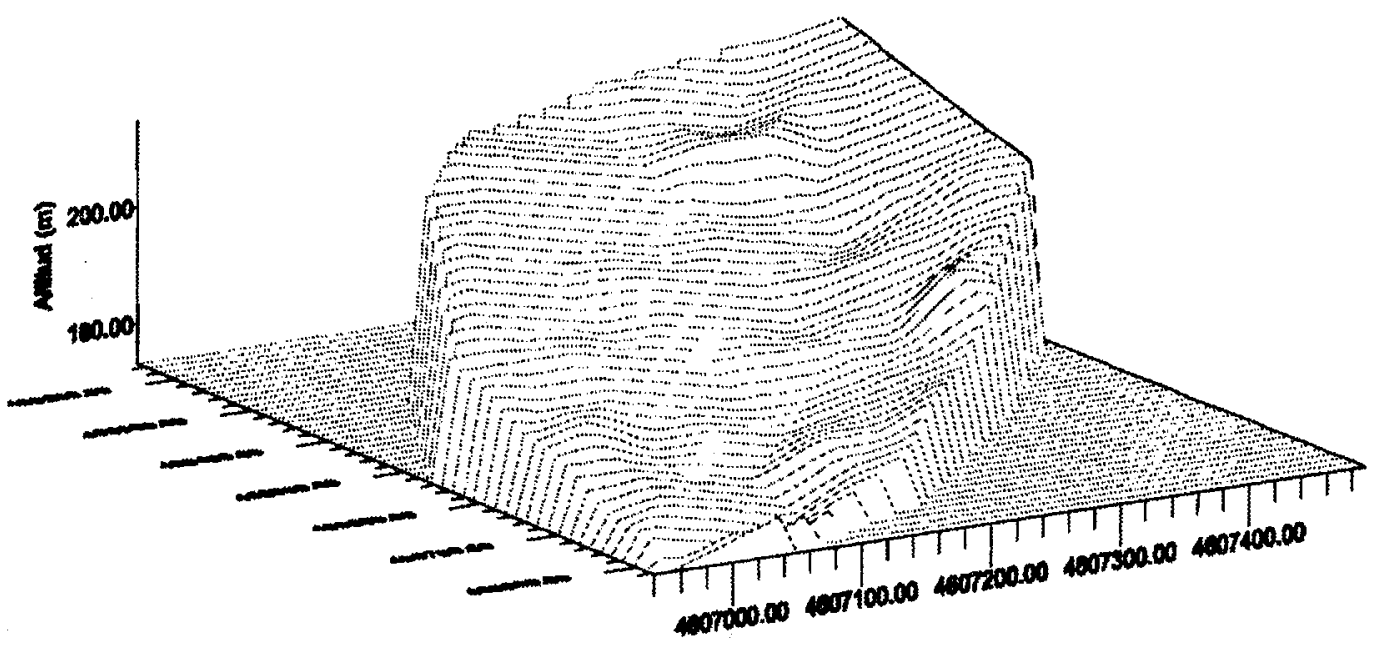

Figura 4. Modelo digital del terreno de la CETM.

Dadas las finalidades de la cuenca, más relacionadas con la interacción suelo-uso agrícola que con el balance estrictamente hidrológico, se mantienen, de forma ininterrumpida y desde la campaña 1993-94, tres parcelas experimentales a escala semiproductiva $(0,3 \mathrm{ha})$ donde se realiza una experiencia continuada de laboreo de conservación: se aplican tres sistemas de laboreo (tradicional, siembra directa y mínimo laboreo) para desarrollar una rotación de leguminosa-trigo-trigo-cebada. Esta parcela está concebida como una herramienta más de la cuenca y de la que se recogen datos de humedad del suelo (TDR) y de efectos sobre porosidad, infiltración, conductividad hidráulica, disponibilidad de nutrientes y contenido de materia orgánica. Las dimensiones de la parcela permiten la realización de ensayos a escala productiva (mecanización, tratamientos y producción).

Tabla 6. Características bidrológicas de la cuenca y equipamiento disponible

\begin{tabular}{lr|ll}
\hline \multicolumn{1}{c|}{ CARACTERÍSTICAS HIDROLÓGICAS } & \multicolumn{2}{c}{ EQUIPAMIENTO } \\
\hline Área de drenaje, ha & 27.13 & Aforo de la cuenca & H flume \\
Longitud del tálweg, $\mathrm{m}$ & 923 & & Sensor de nivel \\
Máxima distancia vertical, $\mathrm{m}$ & 48 & & Trampa de sedimentos \\
Pendiente media tálweg, \% & 5.4 & & Piezómetro \\
Densidad drenaje, canales /ha & 0.037 & & Pluviómetro \\
\hline Tipo de corriente & Continua, efimera & Parcela experimental & Sondas de TDR (0- 90 cm) \\
& & laboreo & Sondas de temperatura \\
\cline { 2 - 4 } Tipo de tálweg & Modificado & Resto de la cuenca & Sondas de TDR \\
& Antropicamente & & Pluviómetros \\
\hline
\end{tabular}

Fuente: Cañameras, et al.,1998 
Desde esta misma época se mantiene una cuarta parcela $(0,15 \mathrm{ha})$ abandonada, donde se simula la no-intervención (ni agrícola ni de cualquier otro tipo). De esta parcela se tienen registros de humedad del suelo entre 0 y $20 \mathrm{~cm}$.

En el área forestal de la cuenca (aproximadamente 1 ha) se instaló una parcela de intercepción pluviométrica, con 5 canales colectores de $1 \mathrm{~m}^{2}$ de superficie, con su correspondiente equivalente en el exterior del área forestal.

\section{Resultados}

\subsection{Caracterización de la cuenca}

Los datos básicos de la superficie del suelo se ha abordado mediante un inventario sistemático de algunos parámetros: contenido de materia orgánica, relación $\mathrm{C} / \mathrm{N}$, $\% \mathrm{CaCO}_{3}$ y granulometría de la tierra fina. Los datos obtenidos se han utilizado para establecer la distribución espacial y las relaciones entre las variables a escala de cuenca. Se ha usado programas de software que, mediante herramientas geoestadísticas (krigging), permiten cartografiar esta distribución (Hereter et al., 2000).

La mayoría $(70 \%)$ de las 106 muestras superficiales $(0-20 \mathrm{~cm})$ analizadas presentan una clase textural franco-limosa. Las restantes se clasifican como francas y franco-arenosas. Se observa una cierta concentración de partículas minerales más finas en dos puntos de la CETM: en la zona de salida del agua de la cuenca y en su área más noroccidental, con pendientes bastante elevadas (Fig. 5). Esta distribución parece ser derivada de dos hechos: las características granulométricas propias del material original, y también de una ligera acumulación de los materiales perdidos en las zonas de exportación (véase más adelante).

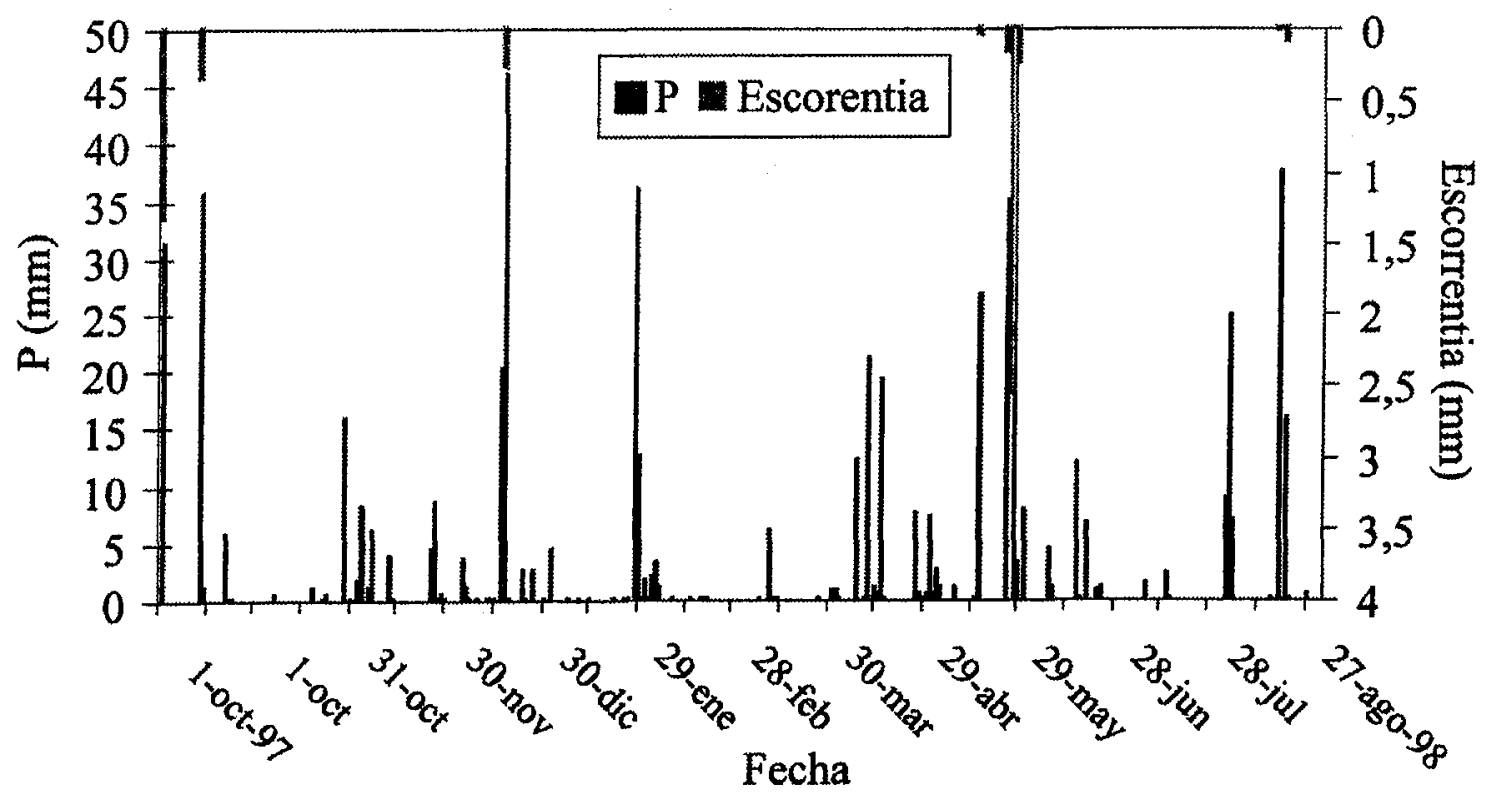

Figura 5. Balance bídrico del ciclo 97-98.

La mayor extensión de la cuenca presenta suelos con 10-25 \% de $\mathrm{CaCO}_{3}$, aunque en algunas zonas, límite NW-SE, el contenido es muy superior (hasta el 40\%). Las 
variaciones naturales del material carbonatado original explican la alta heterogeneidad espacial en este parámetro.

Los contenidos de materia orgánica (M.O.) en el horizonte superficial son los usuales en los suelos cultivados de la zona (Tabla 7). La mitad de las parcelas agricolas tienen contenidos medios en materia orgánica entre 2 y $3 \%$, mientras en una tercera parte presentan 1 - $2 \%$ M.O. La distribución del contenido y la calidad de la materia orgánica y el nitrógeno superficial se relacionan principalmente en los suelos de la cuenca con su tipo de uso. En la zona con suelo forestal se alcanzan los valores más altos, superiores a $4 \%$ de M.O, mientras que los suelos menos orgánicos son las dedicadas desde hace años, más de 10, a olivares y viñedos (Fig. 5).

Tabla 7. Valores medios y parámetros de variabilidad en los borizontes superficiales de los suelos de la CETM según su tipo de uso

\begin{tabular}{lcccccccrcc}
\hline & \multicolumn{4}{c}{ \% materia orgánica } & \multicolumn{3}{c}{$\%$ nitrógeno } & \multicolumn{3}{c}{$\mathrm{C} / \mathrm{N}$} \\
\hline Uso & $\mathrm{N}$ & Media & $\mathrm{Sd}$ & $\% \mathrm{CV}$ & Media & $\mathrm{Sd}$ & $\% \mathrm{CV}$ & Media & Sd & $\% \mathrm{CV}$ \\
\hline Cultivo extensivo & 68 & 2.05 & 0.69 & 33.7 & 0.13 & 0.03 & 23.1 & 9.3 & 3.53 & 38.0 \\
Cultivo arbóreo & 22 & 1.78 & 0.96 & 53.9 & 0.11 & 0.05 & 45.5 & 9.6 & 3.31 & 34.5 \\
Forestal & 14 & 9.09 & 5.33 & 58.6 & 0.28 & 0.11 & 39.3 & 18.2 & 4.57 & 25.1 \\
\hline
\end{tabular}

$\mathrm{N}=$ número de muestras, $\mathrm{Sd}=$ Desviación estándar, $\% \mathrm{CV}=$ Coeficiente de variación.

\subsection{Balance hidrológico de la cuenca}

Durante los ciclos hidrológicos de 1996 a 1998 se estableció el balance hidrológico a escala de la cuenca de acuerdo con la siguiente ecuación, aplicada a intervalos de una semana:

Evapotranspiración neta $=$ Precipitación - Escorrentía - Variación de la reserva,

De estos balances se concluyó que el comportamiento de la cuenca hay que enmarcarlo en el contexto de las pequeñas cuencas de cabecera (elemento de primer orden de la red hidrográfica) con una respuesta inmediata ante los escasos eventos con intensidad suficiente para generar escorrentía. Como era de esperar, presenta un fuerte déficit hídrico, muy variable entre años (por lo menos para el periodo de control) y con periodos muy cortos que generan escorrentía. Ésta se genera como consecuencia de precipitaciones de elevada intensidad, aunque no excepcional (se han observado los efectos erosivos de un evento con un periodo de retorno de 5-6 años). En la Fig. 6 se presenta a modo de ejemplo el balance hídrico del ciclo $1997-98$ y en la tabla 8 , un resumen de los componentes del balance hídrico. Corresponden a dos años consecutivos hidrológicamente muy diferentes, el primero más húmedo (un $31 \%$ más de precipitación que el promedio) y el segundo ligeramente más seco que la media (-13\%). 

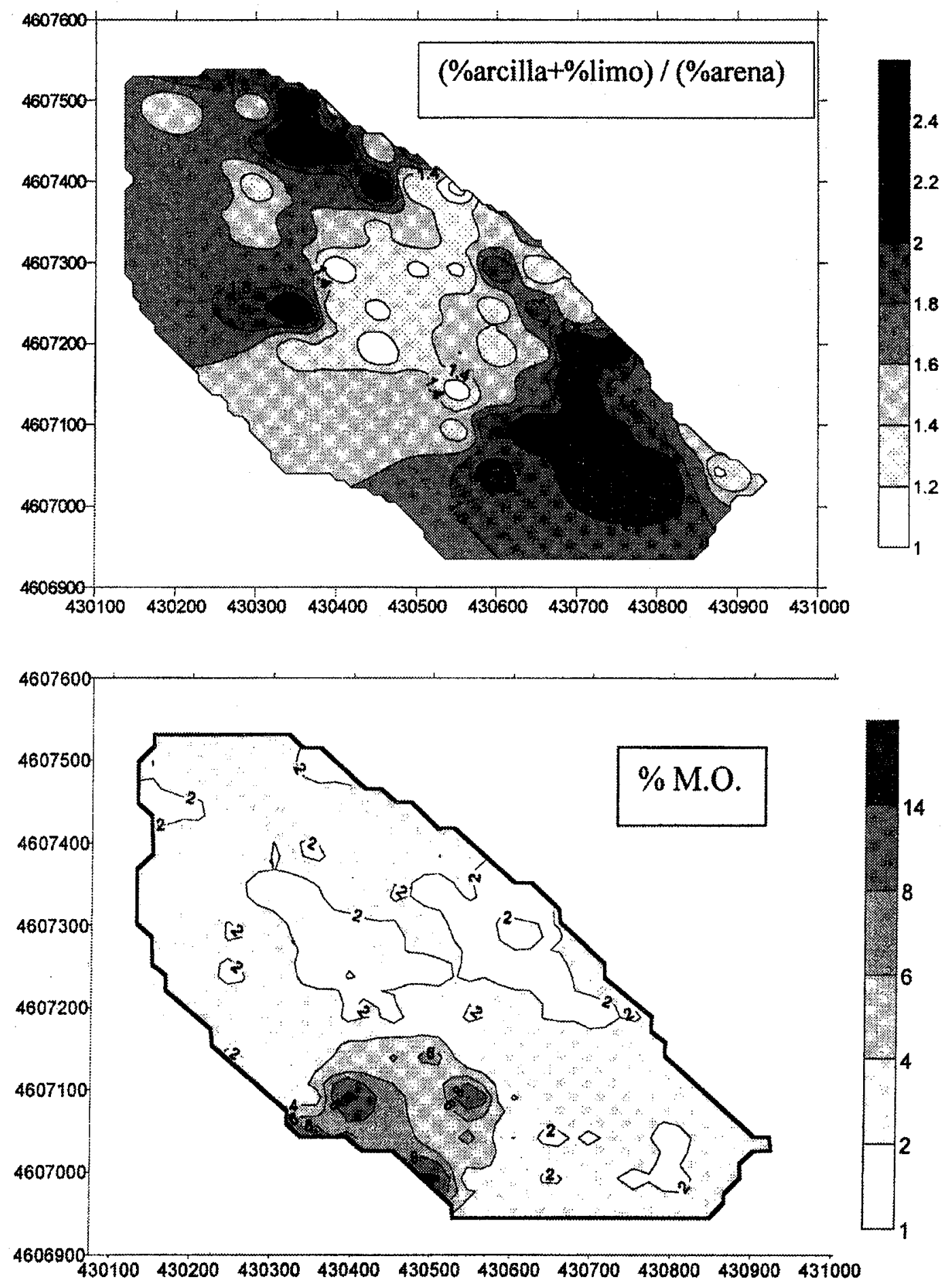

Figura 6. Distribución espacial de las fracciones granulométricas y contenido de materia orgánica del borizonte superficial en la CETM.

\subsection{Medidas de indicadores de procesos de erosión y degradación física del suelo}

La escala de la cuenca hidrológica estudiada ha permitido analizar algunos procesos de erosión, más concretamente de redistribución de material en dirección de la pendiente. En áreas agrícolas mediterráneas, además de la importancia de la irregularidad e intensidad de la precipitación anual, la erosión parece estar ligada a la profundidad del laboreo y a los efectos de la mecanización que afecta a los horizontes subyacentes al labrado (Moussa et al., 1998) . 
Tabla 8. Balance anual de la CETM (ciclos bidrológicos 96 a 98)

\begin{tabular}{ccc}
\hline Términos del ciclo hidrológico & $1 / 10 / 96$ a 30/09/97 & $1 / 10 / 97$ a 30/9/98 \\
\hline Precipitación media anual (mm/año) & 807.4 & 534.9 \\
Escorrentia superficial (mm/año) & $1.7^{*}$ & 3.4 \\
Variación de la reserva edáfica (mm/año) & 82.3 & -7.9 \\
Evapotranspiración real (mm/año) & 723.4 & 539.4 \\
Evapotranspiración real media (mm/dia) & 1.98 & 1.48 \\
\hline
\end{tabular}

* Valor ligeramente subestimado como consecuencia de la pérdida de los datos de dos eventos.

La aproximación al conocimiento de estos procesos se ha llevado a cabo mediante diferentes enfoques. Según Pennock (1997), la concentración de los radioisotopos ${ }^{137} \mathrm{Cs},{ }^{210} \mathrm{~Pb}$ de muestras superficiales es un indicador útil de procesos de redistribución e identificación de erosión del suelo. También se ha analizado la susceptibilidad magnética. El análisis sistemático de los isótopos en la superficie de los suelos de la CETM muestra que existe procesos erosivos a escala de cuenca. Se acumulan en las partículas minerales más finas y presentan la misma distribución espacial que éstas, al igual que la susceptibilidad magnética. Además, los niveles actuales de ${ }^{137} \mathrm{Cs}$ son inferiores a los encontrados en el perfil de referencia, indicando perdidas de suelo (Figs. 7 y 8 ).
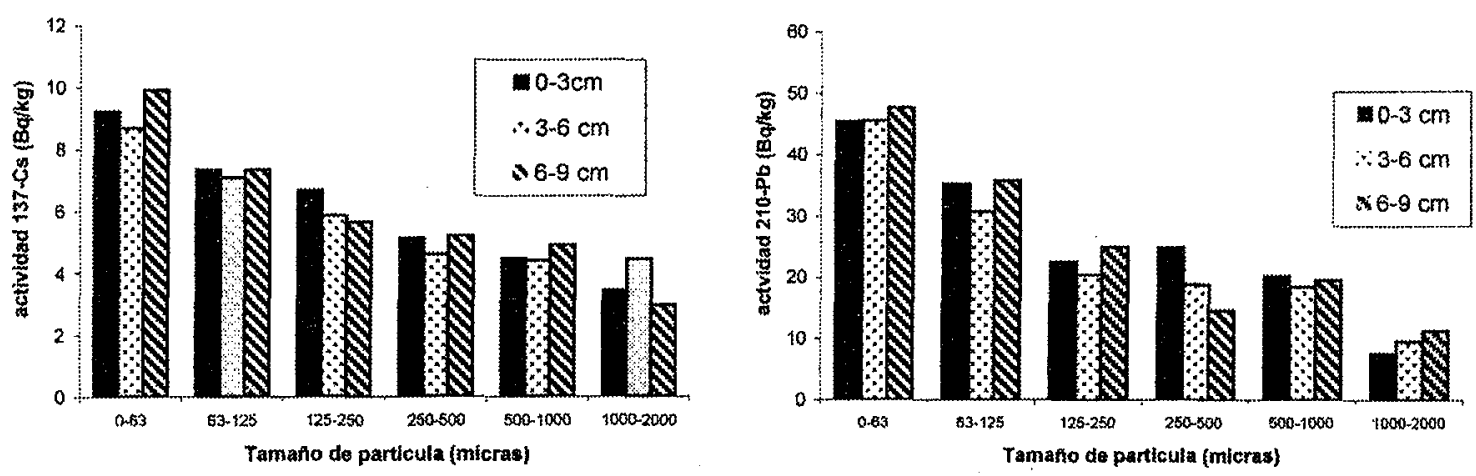

Figura 7. Concentración de radioisótopos en las partículas minerales del borizonte superficial en la cuenca.

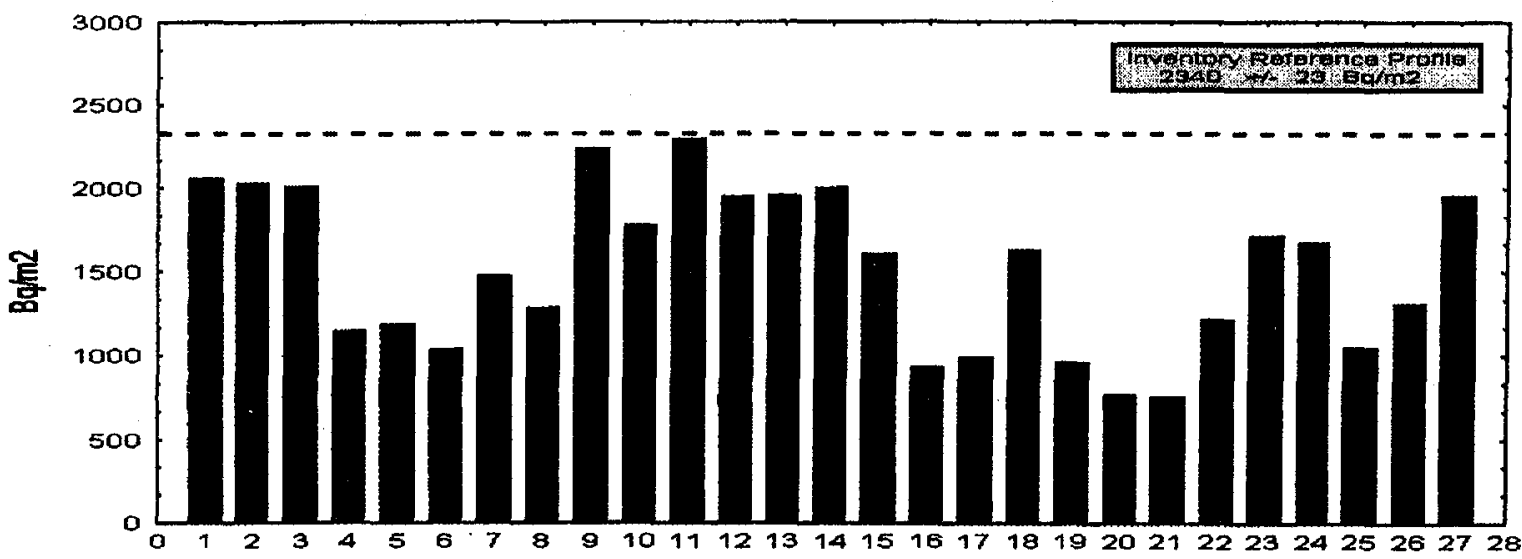

La línea discontinua indica los niveles del perfil de referencia.

Figura 8. Valores de ${ }^{137} \mathrm{Cs}$ en muestras de suelo $(0-30 \mathrm{~cm})$ de la CETM. 
La importancia de la redistribución del material edáfico se ha podido cuantificar y estudiar las áreas mas afectadas a partir de las consecuencias de un evento pluviométrico en el año 1999 (Josa et al. 2000). La intensidad de la lluvia fue muy elevada ya que la precipitación recogida en 24 horas representó el 13,8\% de la media anual, aunque este hecho no es infrecuente en el área mediterránea. En concreto, a partir de datos obtenidos de la estación de Torre Marimon, Páez (1998) calcula un valor del factor $R$, erosividad de la precipitación, de $1662 \mathrm{MJ} \mathrm{mm} \mathrm{ha}^{-1} \mathrm{~h}^{-1}$ año $^{-1}$ (para el año 1995).

La movilización del material edáfico correspondió fundamentalmente al horizonte Ap y se ha estimado en $19 \mathrm{tm} \mathrm{ha}^{1}$. En las zonas afectadas, se crearon rills y gullies que representaron en total $1,7 \mathrm{~km}$ de canales con una densidad de $65 \mathrm{~m}$ de canal por hectárea. Las incisiones eran poco profundas (hasta $15 \mathrm{~cm}$ ) y con un fondo plano entre algunas decenas de centímetros y más de $2 \mathrm{~m}$ de amplitud. En algunos puntos llegan a formarse ollas de erosión de hasta $50 \mathrm{~cm}$ de profundidad.

La cartografía detallada del área afectada, evidenció sin embargo la aparición de forma alterna de zonas exportadoras y de acumulación dentro de la misma cuenca (Fig. 9). Así, la superficie erosionada de forma severa (desaparición completa del horizonte labrado) afecta apenas el $1 \%$ de la superficie de la cuenca y los sedimentos generados ocupan el $1,8 \%$ de la misma.

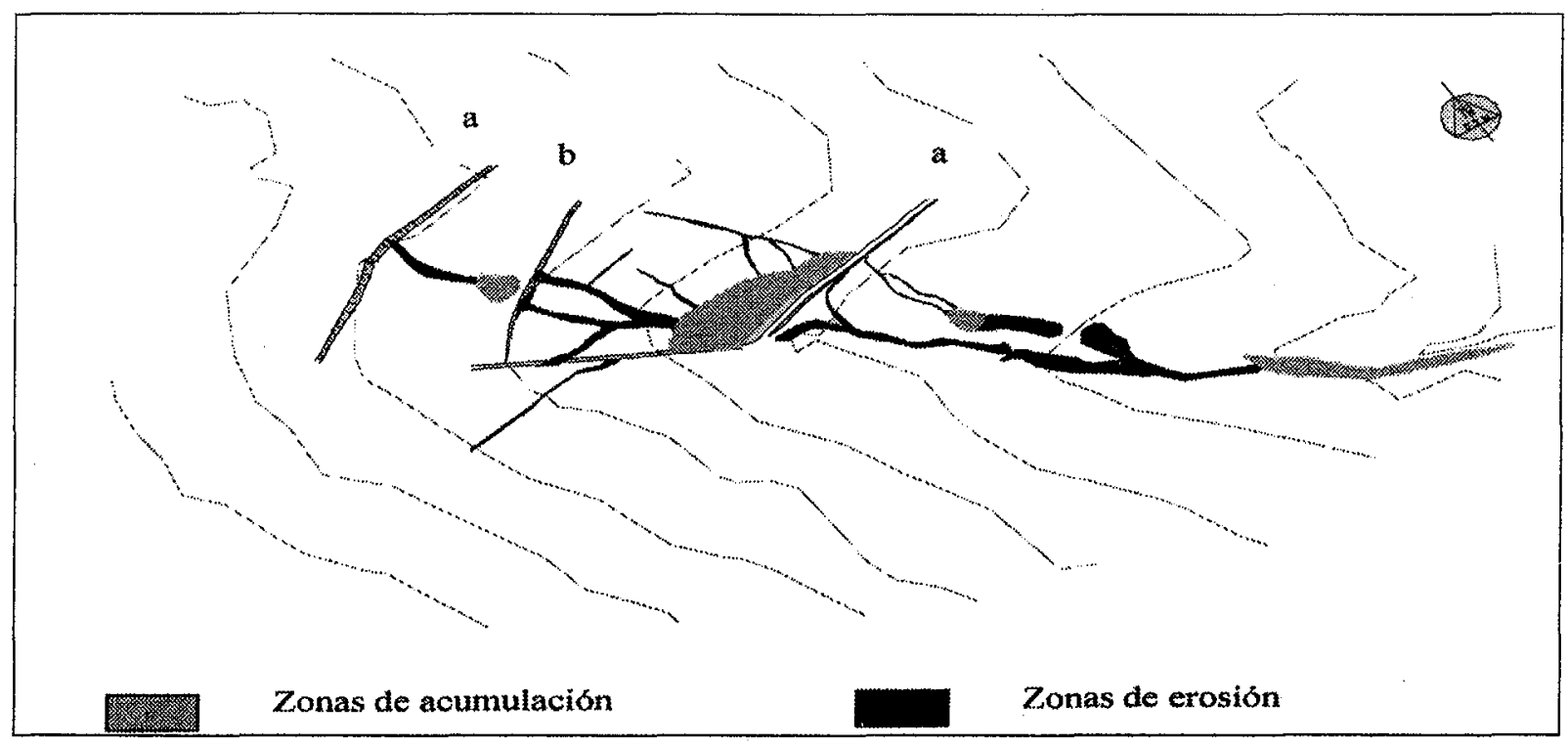

Figura 9. Cartografía de las áreas afectadas por procesos de redistribución del material edáfico superficial en un evento pluviométrico del año 1999.

Paralelamente, se realizó un estudio de parámetros físicos y mecánicos en el perfil edáfico relacionados directamente con el laboreo y su papel en el proceso de erosión. Se analizó las diferencias de conductividad hidráulica, resistencia a la penetración, esfuerzo de cizalla, humedad y porosidad del horizonte Ap y del horizonte subyacente (Ap2) compactado. De esta experiencia, se concluye que las labores agrícolas utilizadas durante un largo periodo, que han propiciado la aparición de la suela de labor, han creado en el perfil edáfico una discontinuidad mecánica e hidrológica a partir de los $15 \mathrm{~cm}$. Se producen cambios significativos de $\mathrm{Ks}$, con 
valores hasta cinco veces superiores en el horizonte labrado, y del comportamiento mecánico, pero no de la porosidad total. Se produce de forma significativa un endurecimiento y un aumento de la resistencia al esfuerzo de torsión en el plano vertical que a la práctica conlleva una discontinuidad que actúa como superficie de desplazamiento del horizonte superficial, si las condiciones son adecuadas.

Aunque se han evidenciado cambios significativos en los indicadores físicos de degradación del suelo superficial, cabe resaltar sin embargo, que la removilización de estas capas no supone un empobrecimiento importante de los parámetros indicadores de su fertilidad química a escala de cuenca. Los cambios horizontales superficiales se atribuyen más a los diferentes usos y gestión antrópica de las parcelas, que pueden explicar las heterogeneidades a niveles de superficies pequeñas, que a una exportación general de nutrientes.

\subsection{Experiencia de los efectos del uso de laboreo de conservación}

Desde el año 1993-94 se dispone en la cuenca de tres parcelas en las que se estudian las consecuencias de la introducción de técnicas de conservación en propiedades físicas y químicas del suelo. La elección y periodicidad de los parámetros controlados responde a su diferente significación temporal y espacial. Los datos analizados hasta el momento se refieren principalmente a los 5-6 ciclos agrícolas posteriores a la introducción de las técnicas de mínimo cultivo y siembra directa, que se comparan con la parcela control en la que se sigue utilizando un laboreo tradicional. El cultivo precedente a la rotación actual (cereal-leguminosa) fue leguminosa durante diversos ciclos agrícolas.

La utilización de las labores de conservación, por lo que se refiere a la evolución de la materia orgánica, afecta al horizonte superficial (hasta $15 \mathrm{~cm}$ ) y en especial los primeros $5 \mathrm{~cm}$ (Dalmau et al., $1999 \mathrm{~b}$ ). Las cantidades totales de materia orgánica y nitrógeno son, después de cinco años de experiencia, significativamente superiores en los suelos con siembra directa a los de la parcela labrada convencionalmente (Fig. 10). La misma tendencia se encuentra cuando se analiza el nitrógeno mineral medio y el fósforo asimilable (Hereter, 1998; Contreras, E. y Llop,1999).

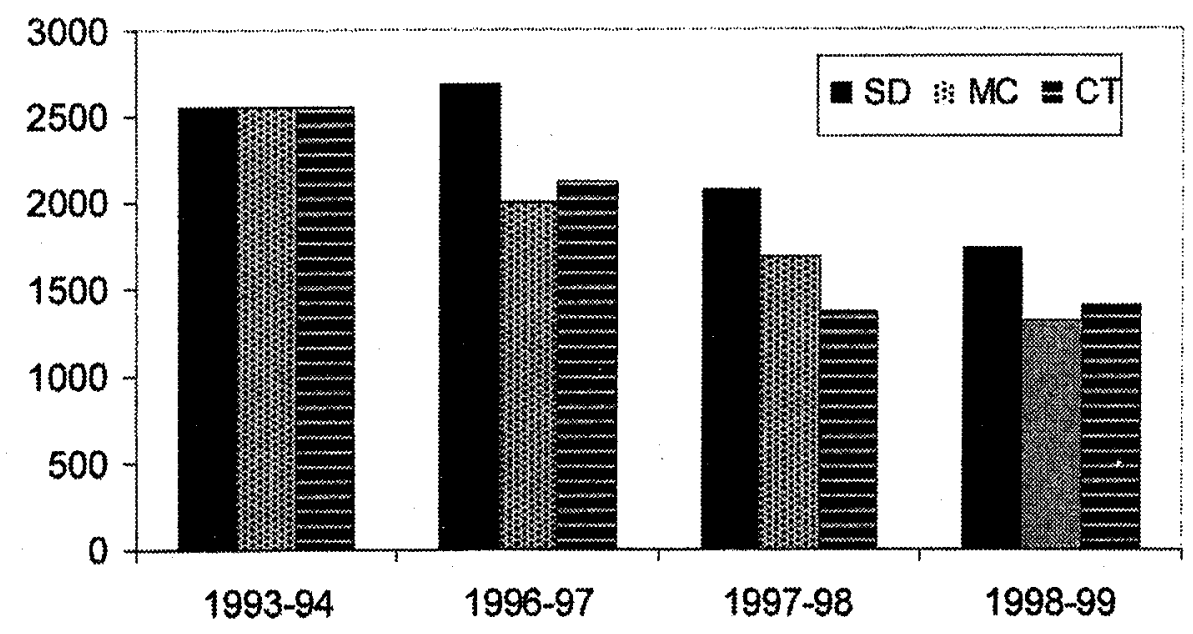

$\mathrm{Kg} \mathrm{N} /$ ha

Figura 10. Contenido de $N$ total en los $0.15 \mathrm{~cm}$ de los suelos de las parcelas con sistemas de conservación. 
El control de los parámetros hidrológicos se ha abordado desde diferentes series de medidas del contenido hídrico, infiltración con el doble anillo y conductividad hidráulica. De lo analizado hasta la fecha parece deducirse que el tipo de trabajo del suelo no modifica substancialmente las características hidrológicas, aunque algunos parámetros si que se ven afectados. En la tabla 9 se presenta los resultados de humedad del suelo (TDR) durante un ciclo vegetativo, en los que se muestra como la utilización de siembra directa provoca un incremento significativo en el contenido de agua del suelo, cuando se compara con los otros tipos de laboreo o su abandono (Josa et al., 1998). Similares resultados se han encontrado en otras campañas (Dalmau et al., 1999 a). Además, existe una tendencia en los suelos menos perturbados a reducir la tasa de infiltración, paralela a un incremento en la densidad aparente. Después de seis años de iniciado el tratamiento, la disminución media correspondía a un $10 \%$ anual (Dalmau et al., 1999 c).

Tabla 9.- Contenido bídrico (1994-95) en los $20 \mathrm{~cm}$ superficiales de las parcelas con diferente sistema de laboreo

\begin{tabular}{ccccc}
\hline $\begin{array}{r}\text { Parcela } \\
\text { experimental }\end{array}$ & Abandonada & $\begin{array}{c}\text { Siembra } \\
\text { directa }\end{array}$ & $\begin{array}{c}\text { Mínimo } \\
\text { laboreo }\end{array}$ & $\begin{array}{r}\text { Cultivo } \\
\text { tradicional }\end{array}$ \\
\hline $\mathrm{m}^{3} / \mathrm{m}^{3}$ & $0.146 \mathrm{~b}$ & $0.177 \mathrm{a}$ & $0.153 \mathrm{~b}$ & $0.146 \mathrm{~b}$ \\
\hline
\end{tabular}

Los valores medios seguidos de letras diferentes indican diferencias significativas al $95 \%$.

\section{Agradecimientos.}

Los autores agradecen la colaboración de T. Martiañez en los trabajos de mantenimiento de las instalaciones de la cuenca.

\section{Bibliografia}

C.E.C. 1985. Soil Map of the European Communities 1:1 000.000. UE. Luxembourg, 124 pág.+ 6 mapas.

CAÑAMERAS, N., DALMAU, L., HERETER, A., JOSA, R. VERDÚ, A.,GIL, E. \& GORCHS, G., 1998. Aproximació a les condicions del moviment de l'aigua en una conca experimental de Torre Marimon. Arxius de l'ESAB. Sèrie V, 1:55-62.

CASAS, A., VILAS, M. \& HELLÍN, C., 1999. VAHMPIRE. Partner 4. Final Report. En: Gallart,F., White,S. y Llorens, P. (ed), Validating bydrological models using process studies and internal data from research basins: tools to assessing bydrological impacts of environmental change. Documento de difusión limitada, Barcelona.

CONTRERAS, E. \& LLOP, N., 1999. Efecte del laboreig en els continguts de matèria orgànica, nitrogen i fòsfor en la superfície del sòl. Trabajo de Final de Carrera de la E.S.A.B., Barcelona, 64 pág + anexos.

D.G.C. 1999. Máximas lluvias diarias en la España peninsular. Ministerio de Fomento, Madrid, 28 pág. + CD-Rom. 
DALMAU, L., HERETER, A., JOSA, R. \& VERDÚ, A., 1999a. VAHMPIRE. Partner 3. Final Report. En: Gallart,F., White,S. y Llorens, P. (ed), Validating bydrological models using process studies and internal data from research basins: tools to assessing bydrological impacts of environmental change. Documento de difusión limitada, Barcelona.

DALMAU, L., HERETER, A. \& JOSA, R., 1999b. Induced changes in soil properties on account of introduction of conservation tillage systems: evaluation on the effects on soil fertility. En Musy,A., Pereira,L.S. y Fritsch,M. (ed.) Emerging technologies for sustainable land use and water management. Proceedings. Lausane, CD-Rom.

DALMAU, L., HERETER, A. \& JOSA, R., 1999c. Reduction of infiltration rate as a consequence of direct sowing. En: J. Bech (ed). Gth International meeting on soils with mediterranean type of climate. Extended Abstracts. Publicacions Universitat de Barcelona. Barcelona, 631-633 pp.

GOMILA FERRANDO, J. M., 2000. Característiques superficials dels sòls d'una conca d'ús agrícola $i$ forestal al Vallès Oriental (Torre Marimon). Trabajo de final de carrera E.S.A.B., Barcelona. 50 pág. + anexos.

HERETER, A., 1998. Soil Organic matter and nitrogen contents after several years of different tillage treatments. En:. XVI World Congress of Soil Science. Summaries Book Vol 1. ISSSAFES. Montpellier. $462 \mathrm{pp}$.

HERETER, A., JOSA, R., SOLÉ, A., VALLÉS, I., COLL, A. \& QUERALT, I., 2000. Variability of topsoil characteristics as indicator of soil material redistribution and soil quality within an agricultural catchment (Torre Marimon, NE Spain). En: Rubio, J.L., et alt. (ed), Man and soil at the tirad millenium. Abstract book. E.S.S.C. Valencia, 243 pp.

I.T.G.E., 1993. Mapa Geológico de España. Escala 1:50.000. Mataró. Segunda Reimpresión. S.G.E.R.M. Ministerio Industria y E., Madrid, 25 pág.+1 mapa.

JOSA, R., VALERO, J. \& ALBORNÀ, S. 1998. Influence of the cultivation system and the relief on the water content of the Ap horizon of land subject to different use. Nutrient Cycling in Agrosecosystems 50: 283-285.

MOUSSA, R., LAGACHERIE, P., VOLT'Z, M., ANDRIEUX,P. \& BOUZIGUES, R., 1998. Hydrological modelling of a farmed mediterranean catchment. En: XVI World Congress of Soil Science. Summaries Book Vol 1. ISSS- AFES. Montpellier. 462 pp.

PÁEZ, S., 1998. Estudio del factor $R$ erosividad de la lluvia en el Vallès Oriental entre los años 1992 y 1997. Trabajo de final de carrera E.S.A.B., Barcelona. 58 pág. + anexos.

PENNOCK, D. J., 1997. Effects of soil redistribution on soil quality: pedon, landscape and regional scales. En Gregorich, E.G. y Carter, M.R. (ed), Soil Quality. Ámsterdam, 167$185 \mathrm{pp}$.

PLAYÀ RABELLÀ, T., 1998. Estudio del factor de erosionabilidad en distintos suelos del Vallés Oriental, sobre un material geológicamente específico. Trabajo de final de carrera E.S.A.B., Barcelona.

QUERALT, L., 1995. Caracterització agroclimàtica $i$ de l'erosivitat de Caldes de Montbui. Trabajo de final de carrera E.S.A.B., Barcelona.

VERDÚ, A.M.C., 1999. Estructura i creixement d'una pineda de pi blanc i pi pinyer a Torre Marimon (Caldes de Montbui). Arxius de l'ESAB. Sèrie V, 3:37-44. 